\title{
Psychological impact of the management of methicillin-resistant staphylococcus aureus (MRSA) in patients with spinal cord injury
}

\author{
Paul Kennedy and Lucy R Hamilton \\ National Spinal Injuries Centre, Stoke Mandeville Hospital, Aylesbury, Bucks, HP21 8AL, UK
}

\begin{abstract}
Background: Management of MRSA infection includes immediate isolation of the patient. Long periods of isolation are considered to be psychologically detrimental, though little information is available about the impact of isolation as an infection control procedure. The purpose of this study is to examine the psychological well-being of spinal cord injured patients who are isolated as a result of being MRSA positive.

Methods: A cross-sectional matched-control study of MRSA positive patients with MRSA negative patients was carried out at the National Spinal Injuries Centre, Stoke Mandeville Hospital, Bucks, England. Sixteen MRSA positive patients, aged 18 to 65, and their matched controls completed a series of questionnaires to measure aspects of psychological impact. The measures used were functional independence, depression, anxiety, and the affective states of anger, vigour, fatigue and confusion.

Findings: The MRSA positive spinal cord injured patients were only significantly more angry than the control group, although these isolated patients scored higher in all measures.

Interpretations: In this spinal cord injured group the difference between the psychological wellbeing of isolated MRSA positive patients and non-isolated MRSA negative patients is not as great as might have been expected. Patients feel that rehabilitation is affected, but the situation may be improved by providing more space and a better view onto the ward.
\end{abstract}

Keywords: psychological impact; methicillin-resistant staphylococcus aureus (MRSA); isolation; spinal cord injury

\section{Introduction}

Methicillin-resistant staphylococcus aureus (MRSA) is a bacterium transmitted via direct person-to-person contact. It is an increasing problem in hospitals today, mainly spread between patients by the hands of caregivers.

The 1995 Communicable Disease Report figures ${ }^{1}$ for Great Britain showed 257 incidences of the three most common strains of MRSA reported by hospitals, an incidence being three or more patients with the particular strain in a month. This suggests that at least 792 patients in a month were MRSA positive, the actual figure probably being much greater than this.

In line with the Infection Control Guide-lines $\left(\right.$ Duckworth $^{2}$ ), patients identified as MRSA positive require immediate isolation whilst they remain positive, and this may be for 2 to 3 months. Management of these patients results in reduced access to a rehabilitation programme and to other patients. All visitors and staff must be gloved and gowned before entering the isolation room and must adopt general infection control procedures.

Correspondence: P Kennedy, Department of Clinical Psychology, National Spinal Injuries Centre, Stoke Mandeville Hospital, Aylesbury, Bucks HP21 8AL, UK
Long periods of isolation are considered to be psychologically detrimental. It has previously been seen that isolation, or perceptual or sensory deprivation, may lead to some abnormal thought or perceptual occurrences. Lilly ${ }^{3}$ experimentally reduced visual, auditory, and tactile input and found that subjects experienced a wide range of sensory illusions. Similar experiences have been reported by prisoners of war and explorers when subject to isolated conditions. ${ }^{4}$ In a laboratory study involving bedrest and social isolation, Downs ${ }^{5}$ found a significant incidence of distorted sensations experienced by subjects after only $2.75 \mathrm{~h}$ in the experimental conditions.

Additional information on altered sensory environments is available from research into hospitalized patients. Easton and Mackenzie ${ }^{6}$ reported that many patients on intensive care units experienced a fluctuating state of consciousness, characterised by fatigue, distraction, confusion, disorientation, agitation, and ultimately, depression. Bolin ${ }^{7}$ stated that altered sensory environments could produce changes in affect, cognition, and perception. Reported effective changes included fear, anxiety, depression, and rapid mood changes. Non-compliance behaviour 
Psychological impact of MRSA

P Kennedy and LR Hamilton has also been noted, including actions contrary to the doctor's instructions and reduced personal motivation. ${ }^{8}$ Changes have been observed to be most extreme in clinical settings due to negative factors such as pain and patients' attitudes to their condition. Moore ${ }^{9}$ reported that hallucinations are often an example of the cortex attempting to arrange what stimuli are available and to find meaning in the environment, thus maintaining arousal and preventing an imbalance in the reticular activating system.

The clinical evidence would suggest that a period of confinement interrupts progress in rehabilitation and may lead to prolonged hospitalization and psychological withdrawal. Research information on psychological aspects of people who are identified as MRSA positive is unavailable, and a recent Medline search (September 1996) failed to identify any research that explored the psychological dimension.

The purpose of this study is to examine the psychological well-being of spinal cord injured patients who are isolated as a result of being MRSA positive, with the hypothesis that isolation will have some impact on these patients.

\section{Method}

Subjects

Sixteen patients were identified who had received a traumatic spinal cord injury, and who have been isolated for at least 2 weeks as a result of being MRSA positive. This group had a mean age of 31.1 years at the time of injury (range 18 to 65 years) and were all male. The group consisted of six tetraplegics, eight paraplegics, and two incomplete injuries. The mean time since injury was 80.8 weeks (range 9 weeks to 17 years).

The control group was matched for age, sex, level of injury, and time since admission or injury.

\section{Materials}

Functional Independence Measure $\left(\mathrm{FIM}^{10}\right)$. This scores 18 items concerning activities of daily living on the amount of help needed by the participant. Beck Depression Inventory $\left(\mathrm{BDI}^{11}\right)$. This scale is widely used in assessing depression and consists of 21 statements exploring affective and somatic aspects of depression. State Anxiety Inventory (STAI-Form $\mathrm{Y}^{12}$ ), measuring state anxiety with 20 statements concerning how people may feel. Profile of Mood States $\left(\mathrm{POMS}^{13}\right)$. This scale is used to measure effect. Words relating to anger-hostility (POMSA), vigouractivity (POMSV), fatigue-inertia (POMSF), and confusion-bewilderment (POMSC) are rated according to how the participant is feeling on that day. A semi-structured interview schedule to collect information specific to the patients' experiences of MRSA induced isolation.

\section{Procedure}

Ethical approval for this cross-sectional study was obtained from the local ethics committee. Between May 1994 and November 1996, 16 patients were identified as MRSA positive who met the criteria of the study, these criteria being that the patient had a traumatic spinal cord injury and had been isolated for at least 2 weeks. At the beginning of the interview the purpose of the study was explained, the participants were reassured about the confidentiality of the information, and were required to sign a consent form. The questionnaires were verbally administered to all of the participants as some who were tetraplegic were unable to fill them in.

Controls matched for sex, age at injury, level of injury, and time since injury or admission were randomly selected from medical files and information concerning the individuals' scores on the questionnaire measures were recorded.

\section{Results}

The scores of the MRSA positive group were compared with the MRSA negative control group using independent $t$-tests. These values and the means and standard deviation of each questionnaire measure are shown in Table 1. The MRSA positive group scored higher than the MRSA negative group in each questionnaire measure. However, the only significant difference between the two groups is that the MRSA group feel more angry, as measured by the POMS Anger-Hostility scale.

The responses of the isolated patients to the semistructured interview showed that $69 \%$ believed their rehabilitation had been adversely affected. Excluding three patients who were on bedrest, and so not yet involved in a rehabilitation programme, increased this figure to $85 \%$ believing their rehabilitation affected by isolation. Isolation affected the mood of $50 \%$ of the group, only one person of which thought themselves to be in a better mood, due to feeling more relaxed. Thirty percent of the respondents thought that isolation helped their adjustment to the injury, whereas $10 \%$ found isolation detrimental to adjust-

Table 1 Means, standard deviations and $t$-tests of each questionaire measure for the MRSA positive and the control groups

\begin{tabular}{lcccc}
\hline & $\begin{array}{c}\text { MRSA } \\
\text { mean }(S D)\end{array}$ & $\begin{array}{c}\text { Cntrl group } \\
\text { mean }(S D)\end{array}$ & $\begin{array}{c}\text { t-value } \\
\text { Significance } \\
\text { level }\end{array}$ \\
\hline FIM & $82.7(21.8)$ & $69.3(23.0)$ & 1.69 & $\mathrm{NS}$ \\
BDI & $16.5(9.9)$ & $12.3(10.7)$ & 1.16 & $\mathrm{NS}$ \\
STAI & $37.8(19.9)$ & $34.2(15.7)$ & 0.57 & $\mathrm{NS}$ \\
POMSA & $12.4(11.7)$ & $4.9(7.1)$ & 2.17 & 0.037 \\
POMSV & $13.9(6.5)$ & $12.1(7.0)$ & 0.76 & $\mathrm{NS}$ \\
POMSF & $7.1(6.4)$ & $5.8(3.9)$ & 0.70 & $\mathrm{NS}$ \\
POMSC & $3.9(5.7)$ & $2.4(4.4)$ & 0.83 & $\mathrm{NS}$ \\
\hline
\end{tabular}


ment. Relationships were not affected for $60 \%$ of the group, and $20 \%$ found that the privacy as a result of isolation helped their relationships. Suggestions made to improve the conditions of isolation included a better view onto the ward, an effective method of calling for assistance, and more space for physiotherapy so that rehabilitation would not be as affected by isolation.

\section{Discussion}

The aim of this study was to examine the psychological well-being of a group of spinal cord injured patients, isolated because of MRSA infection. Denton ${ }^{14}$ suggests that the overall response of a patient, isolated as a source of infection, is one of stress, however, this is not supported by the present study. The main findings here are that the isolated group did not differ significantly from the control group in levels of functional independence, anxiety and depression, although the isolated group did tend to score higher on all measures. Both groups, although not significantly different, did score within the mild to moderate range of depression. In terms of affect, the isolated group varied significantly from the non-isolated group only on the measure of anger. These results suggest that the general psychological well-being of spinal cord injured patients is not significantly affected by isolation. Knowles ${ }^{15}$ suggests that difficulty in attracting the attention of staff leads to frustration and anger. This was a problem mentioned by some of the patients in the semistructured interview and, therefore, improving the system of calling staff may help lessen hostility.

The semi-structured interview showed that the majority of MRSA positive patients believed that isolation had affected their rehabilitation. Although it is encouraging that, as found by Knowles, ${ }^{15}$ some patients had a positive view of isolation, such as having more privacy, more time to think, or feeling more relaxed, every effort should be made to continue with a rehabilitation programme. The situation may be improved for the patients by providing a better view onto the ward and by providing sufficient space for movement around the room and physiotherapy.

It is important to note, however, that these findings may be specific to the spinal cord injured population. The moderately high level of depression found in both spinal cord injured groups may be reflective that the spinal cord injury is the major event, and not the isolation, that accounts for psychological distress. This study was a fairly small pilot study and further investigation needs to be carried out into the psychological aspects of MRSA isolation within other areas of hospitalization and with larger groups of patients. It may be that the tremendous changes patients with a spinal cord injury are experiencing, their age, and the available level of support mitigate the effects of isolation. A study involving a more vulnerable group, for example older adults, may reveal a greater physiological impact of isolation.

\section{Acknowledgements}

The authors wish to extend their thanks to Dr HL Frankel, Mr B Gardner and Mr I Nuseibeh, as well as the patients who willingly participated in this study.

\section{References}

1 Communicable Disease Report Weekly 1996; Volume 6, Number 23.

2 Duckworth GJ. Diagnosis and management of methicillinresistant staphylococcus aureus infection. British Medical Journal 1993; 307: 1049 - 1052.

3 Lilly JC. Mental effects of reduction of ordinary levels of physical stimuli on intact, healthy persons. Psychiatric Research Reports 1956; 5: $1-9$.

4 Solomon P, Leiderman PH, Mendelson J, Wexler D. Sensory deprivation: a review. American Journal of Psychiatry 1957; 114: $357-363$.

5 Downs FS. Bedrest and sensory disturbances. American Journal of Nursing 1974; 74: 435 - 438.

6 Easton C, Mackenzie F. Sensory-perceptual alterations: delirium in the intensive care unit. Heart and Lung 1988; 17: 229-234.

7 Bolin RH. Sensory deprivation: an overview. Nursing Forum 1974: 13: $240-258$.

8 Ziskind E, Jones H, Filante W, Goldberg J. Observations on mental symptoms in eye-patched patients: Hypnagogic symptoms in sensory deprivation. American Journal of Psychiatry 1960; 116: 893 - 900 .

9 Moore T. Making sense of sensory deprivation. Nursing Times 1991; 87: $36-38$

10 Hamilton BB, Granger CV. Guide for the Use of the Uniform Data Set for Medical Rehabilitation. Buffalo, NY: Research Foundation of State University of New York. 1990.

11 Beck AT, Steer ARA. Beck Depression Inventory Manual. San Antonio, TX: Psychological Corporation, Harecourt, Brace and Jovanovich. 1987.

12 Speilberger CD, Gorsuch RL, Lushene R, Vagg PR, Jacobs GA. Manual for State-Trait Anxiety Inventory (Form Y). Palo Alto, CA: Consulting Psychologists Press Inc. 1983.

13 McNair DM, Lorr M, Droppleman LF. Manual for the Profile of Mood States. San Diego, CA: Educational and Industrial Testing Service. 1981.

14 Denton PF. Psychological and physiological effects of isolation. Nursing 3rd Series 1986; 4: $88-91$.

15 Knowles HE. The experience of infectious patients in isolation. Nursing Times 1993; 89: 53-56. 